\title{
Automatic buildings detection using Sobel, Roberts, Canny and Prewwitt detector
}

\author{
Libor Ladányi, Martin Králik ${ }^{1}$
}

\begin{abstract}
This work deals with the possibilities of contemporary automatic identification of objects. Automatic object identification can be done by two computational procedures, namely object detection and object recognition. This work deals with the automatic buildings detection, specifically. Presented detection is performed using the edge detectors, namely Prewwitt, Roberts, Canny and Sobel. The main goal of our work was to automate the device for the detection of hazardous substances in the air, as the detection of hazardous substances is realized by laser-based CBRN (Chemical, Biological, Radiological and Nuclear) stand-off detectors, which evaluate the measured data from the reflected laser beam. In this case, buildings are the most reflective surfaces. In order to detect a building, it is necessary to find a suitable edge detector to be used in further research and serve as a basis for software solution of automatic identification.
\end{abstract}

K e y w o r d s: automatic object recognition, object recognition, buildings recognition, methods based on feature points

\section{Introduction}

Currently, there is a significant implementation of automated processes in almost all scientific and industrial areas. The main reason for this implementation is to increase the efficiency and accuracy of technological processes. A special type of automated technological process is a process that works with automatic object identification. Automatic object identification is a very complex issue, especially in cases where it is necessary to detect and recognize various geometrically complex and very similar objects. In this work, the term automatic object identification represents two parts, namely object detection and object recognition. Object detection in this work represents a set of mathematical operations applied to the images obtained from the camera, in order to highlight objects of interest. Object recognition is the final part of automatic object identification and in this work, it represents a set of mathematical operations in order to assign objects from the analyzed image to known objects saved in the database. This work deals with the detection of objects (buildings) using selected edge detectors and their comparison.

Automatic object identification (AOI) find its applications mainly in areas such as security, road traffic and medicine. In the case of security field, AOI is most often used in airport buildings where two AOI methods are known, facial recognition method and threat detection method. In addition to airport buildings, border crossings are another most frequent place where AOI is used. In the case of road traffic, the AOI applies in particular to the recognition of vehicle registration plate and vehicle type. In the field of medicine, it is possible to use AOR for the DNA damage analysis, or in the Mycobacterium tuberculosis identification system. Another possible application of AOI is its use in the detection of insulators in the power system [1-8].

Automatic object identification can be divided according to the properties of objects into two groups, namely the recognition of stationary objects and the recognition of moving objects. This work is focus on the automatic recognition of stationary objects, specifically buildings. The buildings in our research represent reflecting surfaces for laser beams detecting the presence of hazardous substances in the air. There are currently several methods used in object detection, such as appearance-based methods, geometry-based methods and methods based on feature points. Of the above methods, the methods based on feature points are currently a very frequently used method due to its advantages, which include mainly illumination invariance, viewpoint invariance and scale invariance. In the methods based on feature points, two concepts are important, namely the detector and the descriptor, because the choice of a suitable detector and descriptor can ensure that the above invariance requirements are met. The object detection presented in this work is based on geometry-based methods, because buildings can be classified as relatively geometrically simple objects. [9-10].

The object detection algorithms used in this work are based on edge detectors, specifically the Sobel, Canny, Prewwitt and Roberts edge detectors are used. The task of edge detectors is to determine places in the image with a significant change in brightness intensity. The advantage of edge detectors is that the edges can be considered invariant to changes in illumination and viewpoint [11].

${ }^{1}$ SEC Technologies, s.r.o, Družstevná 771/5, 03101 Liptovský Mikuláš, Slovakia, info@sec-technologies.com, ladanyi@lm.uniza.sk

https://doi.org/10.2478/jee-2021-0039, Print (till 2015) ISSN 1335-3632, On-line ISSN 1339-309X

(C) This is an open access article licensed under the Creative Commons Attribution-NonCommercial-NoDerivs License (http: //creativecommons.org/licenses/by-nc-nd/3.0/). 


\section{Theoretical background}

\subsection{Sobel detector}

The Sobel detector can be characterized as a simple detector of the horizontal and verticality of the edges of the analyzed objects. The Sobel detector is defined by two matrices (masks) with dimensions of $3 \times 3$. The first mask is used to calculate the gradient $G_{x}$ in $x$ direction and the second mask to calculate the gradient $G_{y}$ in $y$ direction. The above masks are defined by the equation $[12]$

$$
\boldsymbol{h}_{x}=\left[\begin{array}{lll}
1 & 0 & -1 \\
2 & 0 & -2 \\
1 & 0 & -1
\end{array}\right], \quad \boldsymbol{h}_{y}=\left[\begin{array}{ccc}
1 & 2 & 1 \\
0 & 0 & 0 \\
-1 & -2 & -1
\end{array}\right]
$$

where $h_{x}$ or $h_{y}$ are also called the Sobel operator. Calculation of gradient components $G_{x}$ and $G_{y}$ is done by $2 \mathrm{D}$ convolution of the Sobel operator and matrix $I$ representing the image, [12]

$$
\boldsymbol{G}_{x}(y)=\boldsymbol{h}_{x}(y) \otimes \boldsymbol{I} .
$$

In the next step, it is necessary to calculate the absolute magnitude $G$ of the gradient and the direction $\Delta$ of the gradient using the following equations, [12-13]

$$
\begin{aligned}
& G=\sqrt{G_{x}^{2}+G_{y}^{2}}, \\
& \Delta=\tan ^{-1}\left(\frac{G_{y}}{G_{x}}\right) .
\end{aligned}
$$

\subsection{Roberts detector}

As in the case of the Sobel detector, the Roberts detector is defined by two matrices (masks), but with dimensions of $2 \times 2$. The Roberts detector is simple and time-saving to calculate, yet it is not used as often as other edge detectors due to its asymmetry. The matrices of Roberts detector (Roberts operator) are defined by equation [11-12], [14]

$$
\boldsymbol{h}_{x}=\left[\begin{array}{cc}
1 & 0 \\
0 & -1
\end{array}\right], \quad \boldsymbol{h}_{y}=\left[\begin{array}{cc}
0 & 1 \\
-1 & 0
\end{array}\right]
$$

Calculation of gradient components $G_{x}$ and $G_{y}$ is realized in the same way as in the case of the Sobel detector, ie by $2 \mathrm{D}$ convolution of the Roberts operator and matrix representing the image. Magnitude of the gradient is defined by (3) and the direction $\Delta$ of the gradient, by

$$
\Delta=\tan ^{-1}\left(\frac{G_{y}}{G_{x}}\right)-\frac{3 \pi}{4} .
$$

\subsection{Canny detector}

Edge detection using a Canny detector is currently one of the most widely used and popular methods [1517]. The Canny edge detector algorithm can be divided into four steps. In the first step the image is smoothed using a Gaussian filter. In the second step, the calculation of the absolute magnitude $G$ in (3) and the direction $\Delta$ (4) of the gradient is realized. Calculation of gradient components $G_{x}$ and $G_{y}$ is realized as a $2 \mathrm{D}$ convolution of the Sobel operator (1) and matrix $\boldsymbol{I}_{\mathrm{f}}$ representing the smoothed image. The third and fourth steps are nonmaximum suppression and thresholding [16].

Image smoothing (filtering) is realized by convolution of a Gaussian matrix (Gaussian averaging operator) and a matrix representing an image. To calculate the Gaussian matrix, it is possible to use the relation for the calculation of the two-dimensional Gaussian function, [18]

$$
G_{\sigma}=\frac{1}{2 \pi \sigma^{2}} e^{-\frac{x^{2}+y^{2}}{2 \sigma^{2}}}
$$

where $\sigma^{2}$ representing the variance. The Gaussian matrix $(5 \times 5)$ can then be written in the following form

$$
\begin{aligned}
\boldsymbol{G} & =\frac{\exp \left(-\frac{\boldsymbol{H}}{2 \sigma^{2}}\right)}{2 \pi \sigma^{2}}, \quad \text { with } \\
\boldsymbol{H} & =\left[\begin{array}{lllll}
2^{2}+2^{2} & 1^{2}+2^{2} & 0^{2}+2^{2} & 1^{2}+2^{2} & 2^{2}+2^{2} \\
2^{2}+1^{2} & 1^{2}+1^{2} & 0^{2}+1^{2} & 1^{2}+1^{2} & 2^{2}+1^{2} \\
2^{2}+0^{2} & 1^{2}+0^{2} & 0^{2}+0^{2} & 1^{2}+0^{2} & 2^{2}+0^{2} \\
2^{2}+1^{2} & 1^{2}+1^{2} & 0^{2}+1^{2} & 1^{2}+1^{2} & 2^{2}+1^{2} \\
2^{2}+2^{2} & 1^{2}+2^{2} & 0^{2}+2^{2} & 1^{2}+2^{2} & 2^{2}+2^{2}
\end{array}\right],
\end{aligned}
$$

giving for $(\sigma=1)$

$$
\boldsymbol{G}=\left[\begin{array}{lllll}
0.0029 & 0.0131 & 0.0215 & 0.0131 & 0.0029 \\
0.0131 & 0.0585 & 0.0965 & 0.0585 & 0.0131 \\
0.0215 & 0.0965 & 0.1592 & 0.0965 & 0.0215 \\
0.0131 & 0.0585 & 0.0965 & 0.0585 & 0.0131 \\
0.0029 & 0.0131 & 0.0215 & 0.0131 & 0.0029
\end{array}\right]
$$

In practice, however, a normalized Gaussian matrix is used, which is defined as follows [18-19]

$$
\begin{aligned}
\boldsymbol{G}_{N}=\boldsymbol{G} \frac{2 \pi \sigma^{2}}{\operatorname{sum}(G)}=\boldsymbol{G} \frac{2 \pi \sigma^{2}}{6.1689} & = \\
= & {\left[\begin{array}{lllll}
0.0030 & 0.0133 & 0.0219 & 0.0133 & 0.0030 \\
0.0133 & 0.0596 & 0.0983 & 0.0596 & 0.0133 \\
0.0219 & 0.0983 & 0.1621 & 0.0983 & 0.0219 \\
0.0133 & 0.0596 & 0.0983 & 0.0596 & 0.0133 \\
0.0030 & 0.0133 & 0.0219 & 0.0133 & 0.0030
\end{array}\right] . }
\end{aligned}
$$




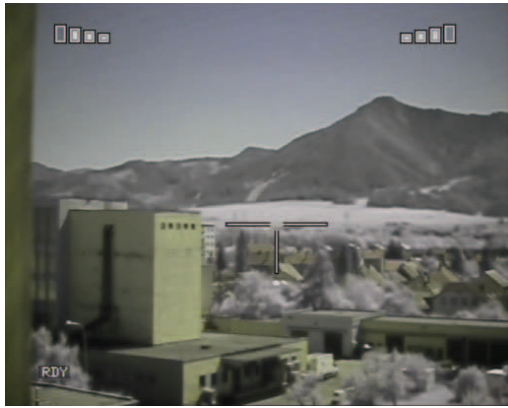

(a)

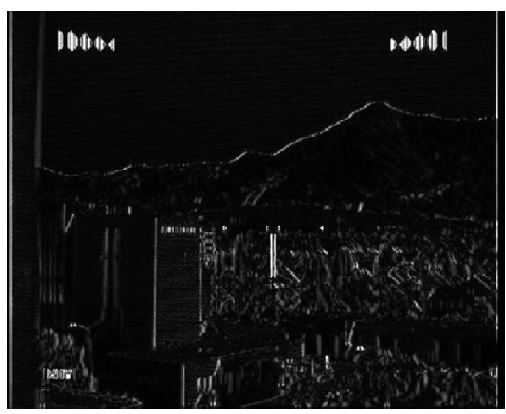

(d)

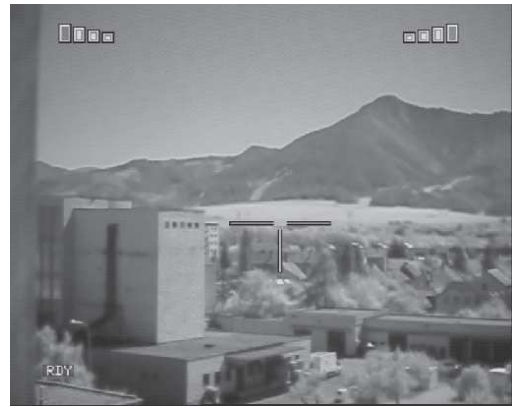

(b)

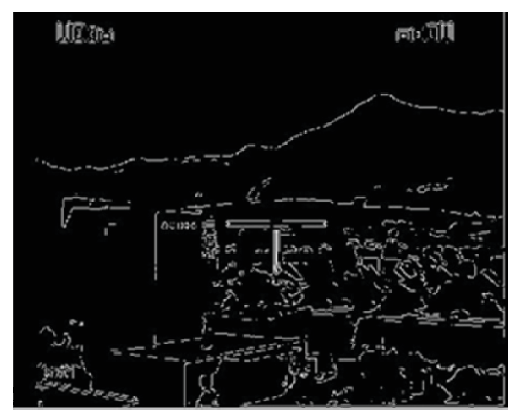

(e)

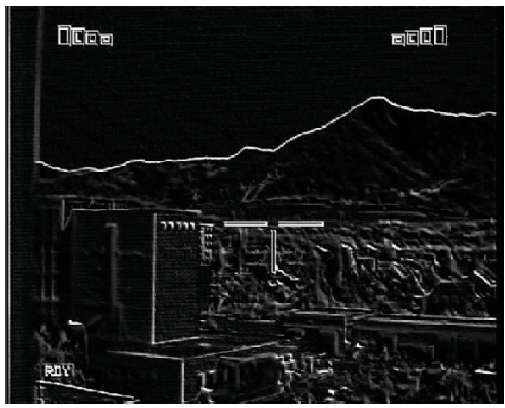

(c)

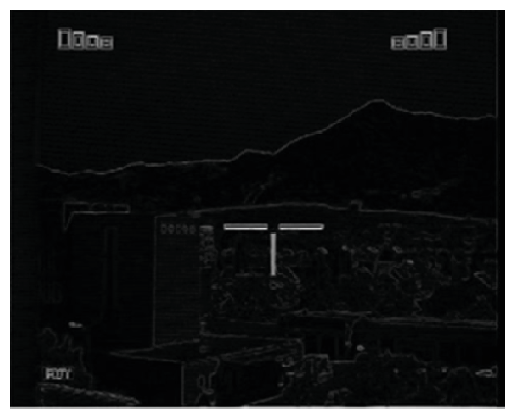

(f)

Fig. 1. (a) - original image, (b) - image converted to grayscale format, (c) - edge detection using Sobel detector, (d) - Prewwitt detector, (e) - Canny detector, and (f) - Roberts detector

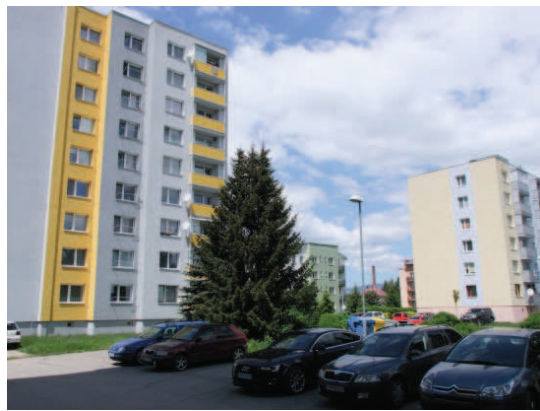

(a)

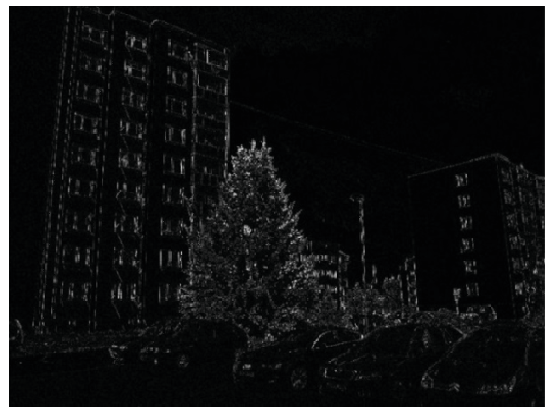

(d)

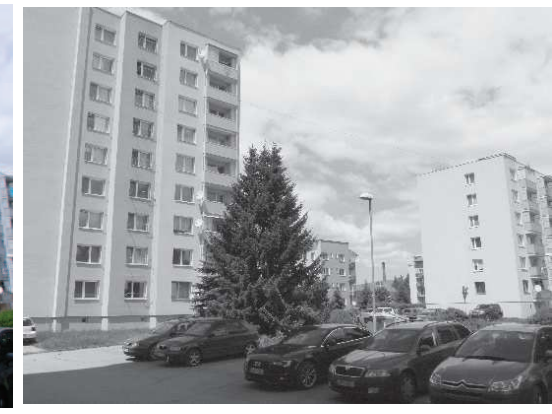

(b)

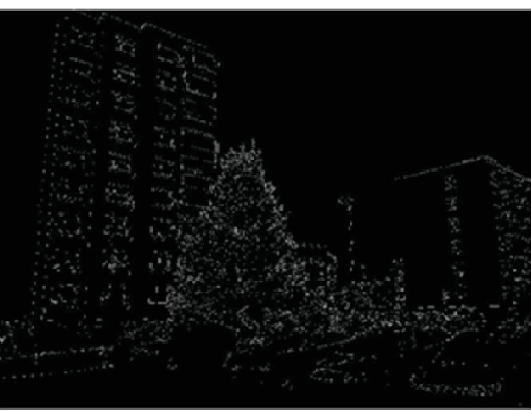

(e)

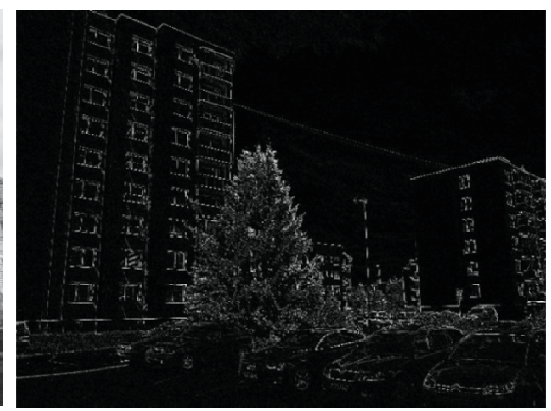

(c)

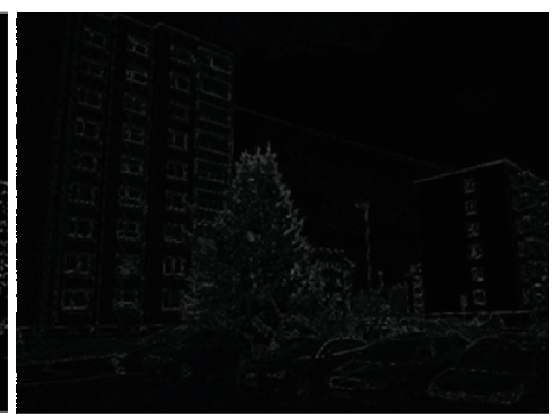

(f)

Fig. 2. (a) - original image, (b) - image converted to grayscale format, (c) - edge detection using Sobel detector, (d) - Prewwitt detector, (e) - Canny detector, and (f) - Roberts detector

\subsection{Prewitt detector}

The computational algorithm of the Prewitt detector is similar to that of the Sobel detector. The matrices (masks) of Prewwitt detector are defined, [12] as

$$
\boldsymbol{h}_{x}=\left[\begin{array}{ccc}
1 & 0 & -1 \\
1 & 0 & -1 \\
1 & 0 & -1
\end{array}\right], \quad \boldsymbol{h}_{y}=\left[\begin{array}{ccc}
1 & 1 & 1 \\
0 & 0 & 0 \\
-1 & -1 & -1
\end{array}\right] .
$$




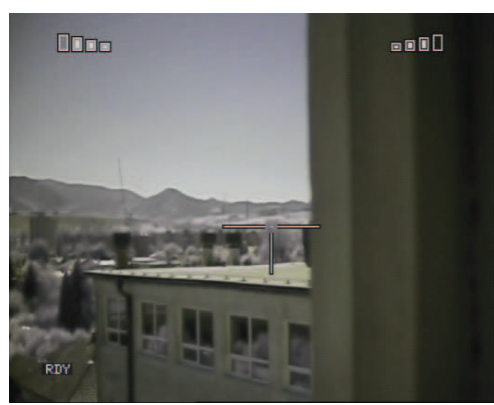

(a)

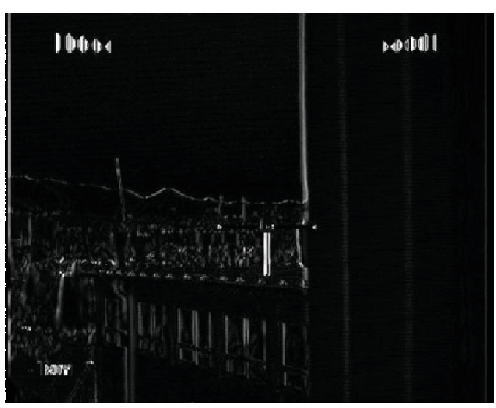

(d)

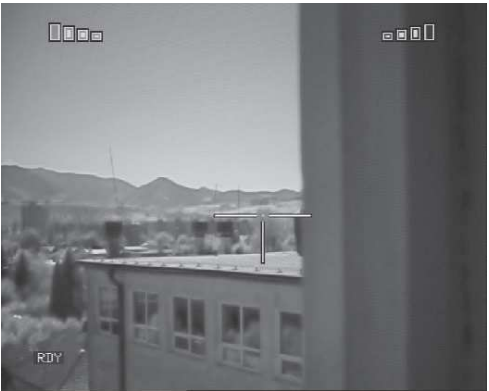

(b)

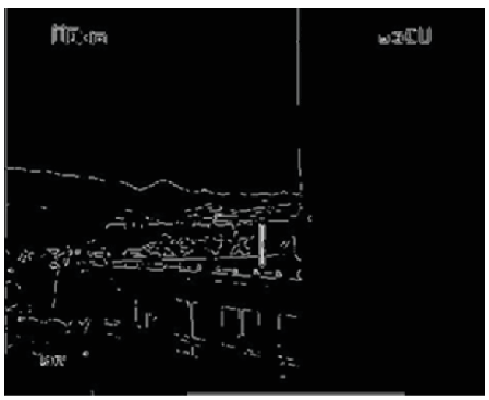

(e)

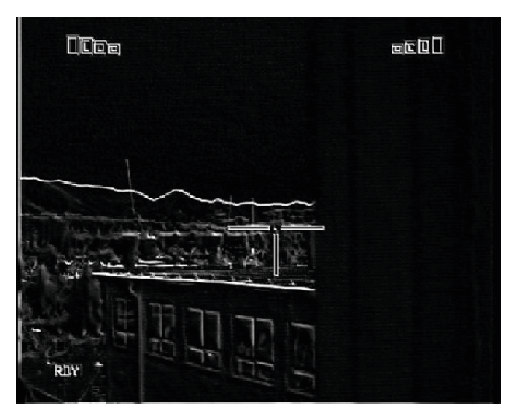

(c)

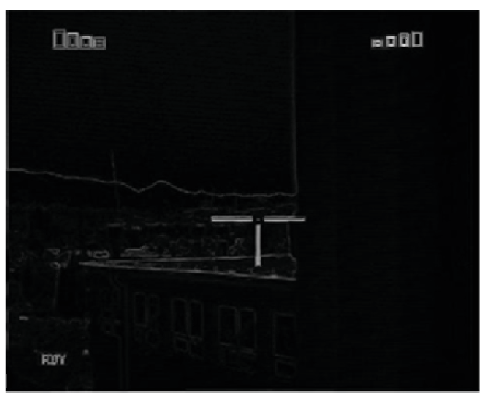

(f)

Fig. 3. (a) - original image, (b) - image converted to grayscale format, (c) - edge detection using Sobel detector, (d) - Prewwitt detector, (e) - Canny detector, and (f) - Roberts detector

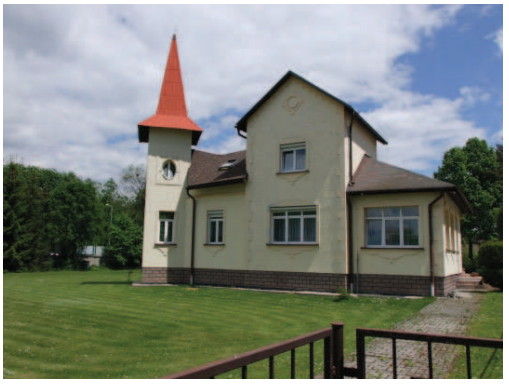

(a)

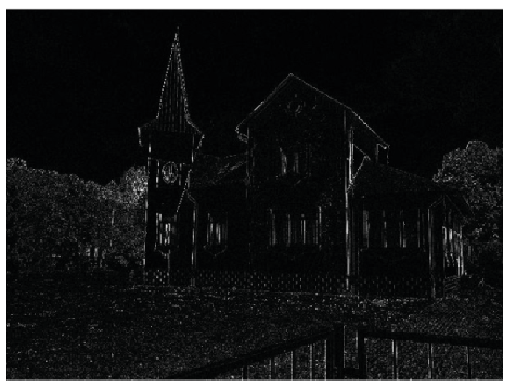

(d)

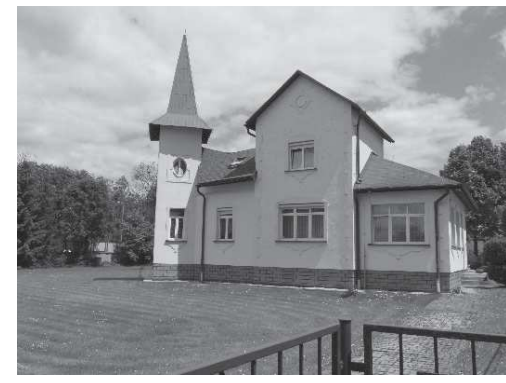

(b)

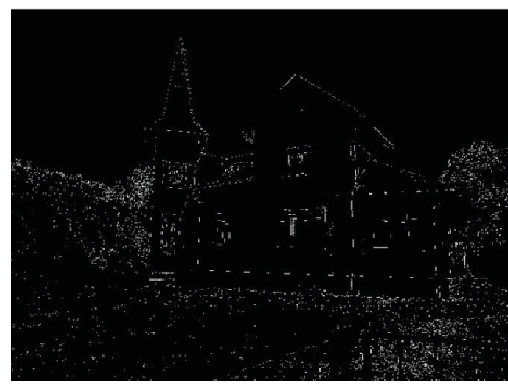

(e)

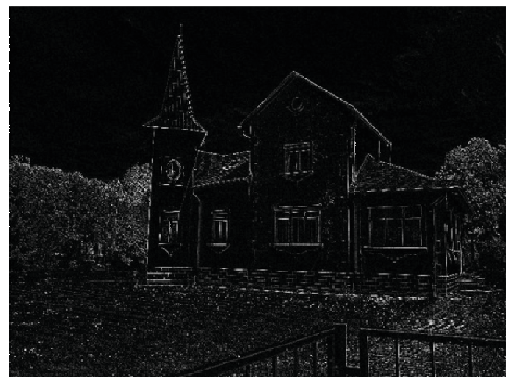

(c)



(f)

Fig. 4. (a) - original image, (b) - image converted to grayscale format, (c) - edge detection using Sobel detector, (d) - Prewwitt detector, (e) - Canny detector, and (f) - Roberts detector

\section{Results and discussions}

We have divided dealing of automatic object identification into two parts, object detection and object recognition. In the first part of our research, we decided to use the methods of the most well-known edge detectors for automatic object detection. To find a suitable edge detector, comparison of Prewitt, Canny, Roberts and Sobel edge detectors was performed.Figures 1-4 show original images of different buildings from different distances us- ing optical zoom and images after application of the presented edge detectors. Of all the presented edge detectors, the Roberts edge detector shows the worst results, while the most satisfactory results are observed using the Canny and Sobel edge detectors. The edge detection in all cases was based on the theoretical foundations given in the chapter describing the theoretical background.

Due to the optimal results of the Canny and Sobel edge detectors, these two edge detectors will be used in the next steps of our research. The Canny edge detec- 
tor is the most time consuming in terms of calculations compared to other detectors published in this work. The computational complexity lies in the fact that the Canny edge detector requires two mathematical parts, namely the convolution of the original image with a Gaussian filter (9) and then the convolution with the Sobel operator (1). Despite the computational complexity, we decided to implement the Canny detector in our software solution, due to its favorable results. For this reason, our next work will be mainly consist in significantly suppressing the computational time required to detect edges using the Canny detector.

Table 1. Parameters of analyzed images

\begin{tabular}{lcccc}
\hline Figure & $1(\mathrm{a})$ & $2(\mathrm{a})$ & $3(\mathrm{a})$ & $4(\mathrm{a})$ \\
Format & BMP & JPG & BMP & JPG \\
\hline Resolution & 720 & 4000 & 720 & 4000 \\
& $\times 576$ & $\times 3000$ & $\times 576$ & $\times 3000$ \\
Bit depth & 24 & 24 & 24 & 24 \\
H-resolution (dpi) & 16 & 72 & 16 & 72 \\
V-resolution (dpi) & 16 & 72 & 16 & 72 \\
Size (MB) & 1.18 & 2.64 & 1.18 & 2.89 \\
\hline
\end{tabular}

\section{Conclusions}

The main goal was to demonstrate the effectivity of four edge detection methods on various images. This is the first step in establishing a suitable method for edge recognition and further research in the field of building and object identification. The crucial parameters of the original selected images are depicted in Tab.1. The selected images were always compressed in a $1 / 3$ ratio before the implementation of the mask for a particular detection method and converted to rgb format. This compression was used to reduce the duration of the entire mask application process for a given image. Based on the achieved results of the analyzed edge detectors, we decided to use two detectors in our further research, namely the Sobel and Canny edge detector, which will form the basis of the software solution for automatic building identification.

\section{Acknowledgements}

This work was supported by the Slovak Research and Development Agency under the project APVV-19-0569.

\section{REFERENCES}

[1] P. Steno, A. Alsadoon, P. W. C. Prasad, T. Al-Dala'in, and O. H. Alsadoon, "A novel enhanced region proposal network and modified loss function, threat object detection in secure screening using deep learning", The Journal of Supercomputing, vol. 77 , pp. 3840-3869, 2021.
[2] S. Lu, B. Wang, H. Wang, L. Chen, M. Linjian, and X. Zhang, "A real-time object detection algorithm for video", Computers \& Electrical Engineering, vol. 77, pp. 398-408, 2019

[3] W. A. Okaishi, A. Zaarane, I. Slimani, I. Atouf, and M. Benrabh, "A Vehicular Queue Length Measurement System in Real-Time Based on SSD Network", Transport and Telecommunication, vol. 22, no. 1, pp. 29-38, 2021.

[4] R. Rosati, L. Romeo, S. Silvestri, F. Marcheggiani, L. Tiano, and E. Frontoni, "Faster R-CNN approach for detection and quantification of DNA damage in comet assay images", Computers in Biology and Medicine, vol. 123, 103912, 2020.

[5] C.-P. Kuok, M.-H. Horng. Y.-M. Liao, N.-H. Chow, and Y.-N. Sun, "An effective and accurate identification system of $\mathrm{My}$ cobacterium tuberculosis using convolution neural networks", Microscopy research and technique, vol. 82, no. 6, pp. 709-719, 2019.

[6] Ch. Xu, B. Bo, Y. Liu, and F. Tao, "Detection Method of Insulator Based on Single Shot MultiBox Detector", Journal of Physics: Conference Series, vol. 1069, 012183, 2018.

[7] C.-J. Li, Z. Qua, S.-Y. Wang, and L. Liu, "A method of cross-layer fusion multi-object detection and recognition based on improved faster R-CNN model in complex traffic environment", Pattern Recognition Letters, vol. 145, pp. 127-134, 2021.

[8] Z. Zhang, P. Chen, X. Shi, and L. Yang, "Text-Guided Neural Network Training for Image Recognition in Natural Scenes and Medicine", IEEE Transactions on Pattern Analysis and Machine Intelligence, vol. 43, no. 5, pp. 1733-1745, 2021.

[9] J. Matas and Š. Obdržálek, "Object recognition methods based on transformation covariant features", 12th European Signal Processing Conference, pp. 1721-1728, 2004.

[10] D. Giveki, M. A. Soltanshahi and G. A. Montazer, "A new image feature descriptor for content based image retrieval using scale invariant feature transform and local derivative pattern", Optik, vol. 131 , pp. 242-254, 2017 .

[11] M. Sonka, V. Hlavac and R. Boyle, Image Processing, Analysis, and Machine Vision, Fourth Edition, Cengage Learning, ISBN 978-1-133-59360-7, 2015.

12] X. J. Jiang and P. J. Scott, Characterization of free-form structured surfaces, Advanced Metrology, Editors X. J. Jiang and P. J. Scott, ISBN 978-0-12-821815-0, pp. 281-317, 2020.

13] J. Rogowska, Overview and Fundamentals of Medical Image Segmentation, Handbook of Medical Image Processing and Analysis (Second Edition), Editor I. N. Bankman, ISBN 978-0-12-373904-9, pp. 73-90, 2009.

14] A. K. Cherri and M. A. Karim, "Optical symbolic substitution, edge detection using Prewitt, Sobel, and Roberts operators", Applied Optics, vol. 28, no. 21, pp. 4644-4648, 1989.

15] S. Das, "Comparison of Various Edge Detection Technique", International Journal of Signal Processing, Image Processing and Pattern Recognition, vol. 9, no. 2, pp. 143-158, 2016.

[16] M. S. Nixon and A. S. Aguado, Low-level feature extraction (including edge detection, Feature Extraction \& Image Processing for Computer Vision, (Third Edition), Editors M. S. Nixon and A. S. Aguado, ISBN 978-0-12-396549-3, pp. 137-216, 2012.

[17] O. R. Vincent, and O. Folorunso, "A Descriptive Algorithm for Sobel Image Edge Detection", Proceedings of Informing Science \& IT Education Conference, (InSITE), vol. 9, 2009.

[18] M. S. Nixon and A. S. Aguado, Basic image processing operations, Feature Extraction \& Image Processing for Computer Vision, (Third Edition), Editors M. S. Nixon and A. S. Aguado, ISBN 978-0-12-396549-3, pp. 83-136, 2012.

[19] S. Khorbotly and F. Hassan, "A modified approximation of 2D Gaussian smoothing filters for fixed-point platforms", IEEE 43rd Southeastern Symposium on System Theory, pp. 151-159, 2011.

Received 22 July 2021 